\title{
Lidil
}

Revue de linguistique et de didactique des langues

\section{Les routines, une catégorie pour l'analyse de discours : le cas des rapports éducatifs}

Routines, a Category for Discourse Analysis: The Case of Reports about Children at Risk

\section{Émilie Née, Frédérique Sitri et Marie Veniard}

\section{(2) OpenEdition}

\section{Journals}

Édition électronique

URL : http://journals.openedition.org/lidil/3939

DOI : $10.4000 /$ lidil.3939

ISSN : 1960-6052

Éditeur

UGA Éditions/Université Grenoble Alpes

Édition imprimée

Date de publication : 30 mai 2016

Pagination : 71-93

ISBN : 978-2-84310-326-1

ISSN : $1146-6480$

\section{Référence électronique}

Émilie Née, Frédérique Sitri et Marie Veniard, « Les routines, une catégorie pour l'analyse de discours :

le cas des rapports éducatifs », Lidil [En ligne], 53 | 2016, mis en ligne le 01 janvier 2017, consulté le 29 octobre 2020. URL : http://journals.openedition.org/lidil/3939; DOI : https://doi.org/10.4000/lidil.3939

(C) Lidil 


\title{
Les routines, une catégorie pour l'analyse de discours : le cas des rapports éducatifs
}

\author{
Émilie Née*, Frédérique Sitri** et Marie Veniard***
}

\begin{abstract}
RÉSUMÉ
Nous situant dans la perspective d'une phraséologie étendue, nous proposons de définir les routines comme l'articulation entre un format lexico-syntaxique plus ou moins figé et des fonctions textuelles ou discursives. Nous souhaitons dans cet article proposer une exploitation de la notion de «routine» dans le cadre de l'analyse de discours en montrant comment les patrons repérés par leur fréquence peuvent être mis en relation non seulement avec des fonctions textuelles ou rhétoriques mais également avec des déterminations interdiscursives - fonctions et déterminations que recouvre la catégorie du genre. La notion de routine constitue donc une catégorie d'analyse opératoire pour une analyse de discours soucieuse d'appuyer l'interprétation sur des formes. Nous illustrons notre propos par deux exemples tirés de rapports éducatifs rédigés par des éducateurs spécialisés dans le champ de la protection de l'enfance.
\end{abstract}

\section{ABSTRACT}

Considering phraseology as emcompassing large and semi-fixed units, we investigate and define in this paper "writing routines", a unit articulating fixed or semi-fixed lexicogrammatical patterns and textual or discursive functions. We claim that "routines" can become a descriptive category along with others in discourse analysis since they are genre-related. Indeed, frequent patterns or lexical bundles correlate not only with textual or rhetorical functions but also with dialogismbased constraints, all of these determinations being encapsulated in the notion of "genre". We consider routines as a workable category for a discourse analysis based on linguistic evidence. We illustrate our claim with two examples extracted from reports written by social workers in the field of child protection.

* Céditec, Université Paris-Est Créteil Val-de-Marne.

** Modyco, Université Paris Ouest Nanterre La Défense, CNRS.

*** EDA, Université Paris Descartes. 
Alors que l'analyse du discours s'intéresse depuis longtemps aux phénomènes de répétition et de figement, qui représentent un mode d'entrée privilégié dans les discours, cette discipline n'a pas constitué en objet d'étude la notion de «routine», pourtant courante dans des travaux anglo-saxons relevant de l'analyse conversationnelle et de la linguistique fonctionnaliste. Nous souhaitons dans cet article expliquer comment cette notion peut se révéler une catégorie opératoire pour l'analyse du discours, en l'articulant à celle de «genre». Dans une démarche qui se veut à la fois épistémologique, méthodologique et descriptive, nous proposerons une modélisation de cette notion permettant de penser l'articulation entre phénomènes phraséologiques et déterminations discursives propres aux genres. Cette modélisation est appuyée à une méthodologie de la mise au jour des séquences préfabriquées au moyen des outils d'exploration textométrique. Notre réflexion sur le rôle des routines est nourrie par les résultats d'une recherche en cours sur des rapports éducatifs produits dans le cadre de l'enfance en danger.

\section{La notion de « routine» en sciences du langage}

La notion de «routine» est développée dans des approches linguistiques qui travaillent dans des paradigmes différents de ceux de l'analyse du discours (plus loin, AD), le plus souvent dans la continuité des «routines conversationnelles» (Coulmas, 1981). Dans le modèle développé par Berrendonner et le «groupe de Fribourg» (2012), les routines correspondent à des séquences d'actions énonciatives, qui peuvent être intra-périodiques ou transpériodiques (Groupe de Fribourg, 2012, p. 224) : ainsi, les pseudo-clivées interviennent dans différents types de configurations ou routines comme les réponses-échos, les routines d'énumération, les routines à fonctions contrastives...; ainsi encore les insertions parenthétiques sont décrites comme des routines dans Berrendonner (2008). Si les structures ainsi dénommées se caractérisent par leur caractère ritualisé et leur dimension praxéologique (Corminboeuf, 2013), elles présentent une grande diversité de nature et ne semblent pas consister systématiquement en une suite d'unités discrètes susceptible d'une description formelle. On est ici dans un cadre théorique, différent du nôtre, qui envisage le discours comme «l'ensemble des matériaux sémiotiques mis en œuvre par les partenaires d'une [...] interaction [verbale]» (Groupe de Fribourg, 2012, p. 21). Dans le cadre de la linguistique de corpus, les travaux portant sur des écrits scientifiques (Hyland, 2010; Tutin, 2013; Sandor, 2007; Swales, 
1990) s'intéressent de plus en plus aux «routines rhétoriques» propres à ces écrits : les routines sont associées à des stratégies rhétoriques propres au genre de l'écrit scientifique ( «la démarcation par rapport aux pairs», «la présentation de la problématique de recherche», «la filiation scientifique» dans Tutin, 2013, p. 38). Du point de vue formel, ces routines relèvent d'un schéma phrastique : «elles correspondent à des énoncés complets, construits autour d'un prédicat, et mettent en jeu un lexique varié» (Tutin, 2013, p. 36). D'un point de vue méthodologique, le repérage de ces routines s'inscrit dans une perspective fonctionnelle (au sens de Halliday) qui associe à une fonction rhétorique une modélisation sémantique inspirée de Fillmore et fait appel à des outils de Traitement automatique du langage (voir ici-même Tutin et Kraif).

En analyse du discours, la notion de «routines» ne constitue pas à notre connaissance une catégorie descriptive en tant que telle, mais elle est cependant évoquée, en relation étroite avec la question du genre Ainsi, pour Maingueneau (2004), elle participe à la caractérisation des genres «routiniers» qui, par opposition aux genres «auctoriaux», recouvrent des écrits dont «les paramètres qui les constituent résultent [...] de la stabilisation de contraintes liées à une activité verbale qui s'exerce dans une situation sociale déterminée» (p. 110) et dont les caractéristiques s'imposent à une collectivité de scripteurs ${ }^{1}$. KriegPlanque, de son côté, souligne les déterminations discursives qui régissent les régularités syntaxiques et met en relation les «routines d'écriture» dans les écrits institutionnels avec des régularités génériques ( «l'appartenance [d'un texte] à un genre surdétermine la syntaxe», Krieg-Planque, 2013, p. 108). L'appellation «routines d'écriture» signale que les routines sont envisagées ici non seulement comme des observables langagiers mais aussi comme des processus de production des textes : c'est également le point de vue adopté par BrancaRosoff dans ses travaux sur les écrits des peu lettrés (comptes rendus de comités de surveillance au moment de la Révolution française et

1. Même s'il propose dans la suite de l'article de dépasser cette opposition, le critère retenu pour classer les genres est bien celui de leur position sur l'axe routinisation/créativité, critère qui opère même à l'intérieur de la classe genres routiniers : «À l'intérieur de ces genres routiniers, on peut définir une échelle : d'un côté les genres totalement ritualisés, qui laissent une marge de variation minime (actes juridiques, par exemple), de l'autre ceux qui, à l'intérieur d'un script peu contraignant, laissent une grande part aux variations personnelles.» (Maingueneau, 2004, p. 110) 
lettres de soldats durant la guerre de 14-18; voir Branca-Rosoff, 1990 ; 1997 entre autres). Elle considère en effet que les «formulaires» qu'elle identifie dans certaines parties du texte (ouverture /clôture, description...), qui sont soit des blocs figés, soit des «formes discursivement proches caractérisées par le recours à une famille de formes syntaxiques de fortes restrictions lexicales» participent à «la mise en place de "routines" permettant d'écrire». La prise en compte du point de vue du scripteur est également centrale pour les chercheurs qui travaillent, aux marges de l'AD et de la sociolinguistique, sur les écrits «routiniers» du travail (Delcambre, 1993; Pène, 1994) en soulevant la question de l'émergence de la subjectivité et de la créativité dans ces formulations routinières.

Ainsi, les travaux dans le champ de l'AD inscrivent la question des routines dans une perspective non seulement fonctionnelle et rhétorique, mais également sociale et professionnelle, en relation forte avec une conception bakhtinienne du genre comme produit par une sphère sociale d'activité. Ils s'intéressent peu cependant - mis à part la description précise que propose Branca-Rosoff des «formulaires» - à la caractérisation formelle des routines et à leur repérage dans les discours.

Cette dimension formelle est au contraire centrale pour les recherches sur le discours politique menées au sein du Laboratoire de Saint-Cloud qui, sans thématiser à proprement parler la question des routines, visent à rendre compte de manière systématique des phénomènes de répétition et de figement d'ordre discursif. Les travaux menés dans ce cadre, à l'intersection de l'analyse de discours et la statistique textuelle, mettent en place la notion de segment répété (Salem, 1987), qui permet de saisir les effets de «langue de bois » (Fiala, 1987; Fiala, Habert, Lafon \& Pineira, 1987). Cette conjonction disciplinaire a également beaucoup développé le recours aux cooccurrences ${ }^{2}$ (dont le calcul remonte à Lafon, 1981; voir aussi Heiden \& Lafon, 1998; Martinez, 2003; Mayaffre, 2008a, 2008b, etc.), qui permet de dépasser la contrainte de successivité imposée par le segment répété et de saisir ainsi des formes plus lâches. Ces recherches adoptent cependant une acception très large de la notion de genre : il n'y a pas vraiment de dis-

2. Dans une perspective lexicographique, on peut aussi citer des travaux tels que ceux de Gorcy, Martin, Maucourt \& Vienney (1970), concernant l'extraction des syntagmes pour l'élaboration du TLF ou, en Grande-Bretagne, pour le COBUILD, ceux de Krishnamurthy (2004). 
tinction entre «genre» et «formation discursive» ou «sphère d'activité» (Fiala, Habert, Lafon \& Pineira, 1987, p. 77). Le discours politique et le discours administratif sont ainsi considérés comme des genres. Par ailleurs, les approches textométriques attribuent à des unités telles que les segments répétés un statut d'unités constitutives des textes, caractéristiques des genres, tandis que le calcul des segments répétés constitue plutôt pour nous une voie d'entrée dans le corpus, une mise en avant de points saillants du discours.

C'est en nous inspirant de ces différents travaux que nous proposons une modélisation de la catégorie de «routine» articulée aux déterminations génériques, susceptible d'en faire un instrument d'analyse et de description des discours. Ces propositions sont adossées à l'analyse d'un corpus de textes représentant un genre professionnel, le rapport éducatif, sur lequel nous travaillons depuis une dizaine d'années ${ }^{3}$. Adressés au juge, mais susceptibles d'être lus par la famille, ces écrits visent à décrire et à caractériser une situation familiale afin de préconiser la mise en place, la suspension ou la poursuite d'une mesure éducative. Rédigés par le(s) éducateur(s) référent(s) de l'enfant, ils sont discutés en réunion et relus par le chef de service, qui rédige parfois la conclusion. Ces textes sont régis par des contraintes rédactionnelles plus ou moins explicites (il existe des guides de rédaction, en ligne), et pris dans un interdiscours multiple : on peut saisir les traces de ce que nous avons appelé un discours «psy», très présent dans les catégorisations des comportements («mère fusionnelle» par exemple), mais aussi des représentations propres au discours éducatif comme la valorisation du progrès (voir plus loin l'analyse de la routine «et peut») ou l'importance du cadre (voir l'analyse de «dans le cadre»). On le voit, le genre

3. Nos recherches sur les rapports éducatifs dans le champ de la protection de l'enfance ont débuté en 2005 avec la formation du Groupe Signalement autour d'André Collinot (Université Sorbonne Nouvelle - Paris 3). Elles se sont poursuivies avec la réponse à un appel d'offre de l'Observatoire national de l'enfance en danger (ONED), puis dans le cadre du projet innovant Approche discursive et génétique des brouillons (2010-2012, <http://syled. univ-paris3.fr/projet_innovant/ADGB2008/>) et de l'ANR Écritures (20122015). Ces deux derniers projets ont intégré une perspective génétique, articulant l'analyse des rapports finaux à l'analyse des brouillons et des états successifs de rédaction de ces mêmes rapports. Les analyses abordées dans cet article portent sur ces deux ensembles de données réunies en corpus distincts. Ces corpus peuvent être consultés en ligne sur le lien suivant : <www. univ-paris3.fr/corpus-du-projet-98190.kjsp?RH=1295620557102>. 
du rapport éducatif ne peut être associé à sa seule visée pragmatique articulée à une fonction sociale (évaluer et préconiser). Nous situant dans la filiation de Bakhtine, relayée entre autres par Bronckart (1997), nous concevons le genre comme une catégorie dynamique et hétérogène, en relation avec d'autres genres au sein d'entités plus larges que sont les sphères d'activité ou les formations discursives (Mellet, Rinck \& Sitri, 2013). Si le genre constitue bien une entité «biface» (BrancaRosoff, 1999) articulant matérialité langagière et déterminations extralangagières, les déterminations qui pèsent sur le genre sont multidimensionnelles, ce que l'on retrouve naturellement dans l'approche des routines que nous proposons.

\section{Propositions pour une modélisation de la catégorie de la «routine $»^{4}$}

Deux postulats caractérisent donc notre approche : une perspective formelle de type phraséologique et une approche d'analyse de discours prenant en compte non seulement les visées pragmatiques liées à la fonction du genre mais également les déterminations interdiscursives qui, selon nous, participent (également) à sa caractérisation.

Du point de vue formel, notre objectif est d'identifier des séquences plus ou moins figées qui peuvent être mises en relation avec des fonctions ou des déterminations textuelles ou discursives, et dont la répétition signale qu'elles sont le support de «routines» discursives. Le corpus analysé comporte 22 textes ${ }^{5}$ (44 178 occurrences et 4876 formes). Nous situant dans une conception élargie de la phraséologie outillée par la statistique textuelle, nous avons eu recours pour le repérage de ces séquences aux outils textométriques via le logiciel de textométrie, Le Trameur, conçu par Fleury (2007). Partant de l'index lexical (ou index hiérarchique) ${ }^{6}$ et de l'inventaire hiérarchique des segments répétés du corpus (Lebart \& Salem, 1994, p. 63-69; voir tableau 1 en

4. Le paragraphe qui suit reprend de manière synthétique les propositions formulées dans Née, Sitri \& Veniard (2014).

5. L'orthographe et la typographie des textes originaux ont été conservées telles quelles. Tous les noms propres ont été anonymisés.

6. Terme issu de la statistique lexicale, l'index lexical ou hiérarchique est la liste des formes du corpus, rangées par ordre de fréquence décroissante (voir aussi Lebart \& Salem, 1994, p. 52). 
annexe), nous avons laissé de côté les expressions lexicales renvoyant à des realia propres au domaine («assistante familiale», «juge des enfants») et les associations déterminant/préposition («de la», «à son») pour explorer des séquences remarquables par leur fréquence du type «nous avons», «sur le groupe», «des difficultés», «il peut»... À l'aide des concordances et des calculs de cooccurrence, associés à un retour au texte, nous avons mis en évidence des unités phraséologiques dépassant le cadre du syntagme et de nature multidimensionnelle, car elles peuvent être constituées d'unités hétérogènes. Nous avons proposé d'appeler «patrons » les unités identifiées et nous en proposons la définition suivante :

Moules syntaxiques ou séquentiels avec une combinatoire lexicale plus ou moins restreinte, les patrons peuvent être de l'ordre du syntagme ou de la proposition (patron syntaxique), voire avoir un empan interpropositionnel (patron séquentiel). Ils peuvent comporter des places qui ne sont pas toujours toutes actualisées. Nous posons l'existence d'un continuum entre des séquences très figées et d'autres qui le sont moins. Le moindre degré de figement est représenté par des associations notionnelles ${ }^{7}$.

Dans une perspective d'analyse de discours, nous avons mis en relation les patrons identifiés avec des fonctionnements discursifs se déployant à différents niveaux. Ainsi, le patron «être dans », qui donne lieu à des réalisations comme il peut être dans la dissimulation de ses devoirs ou des mots à faire signer ou $T$ est en recherche affective de la part de sa mère, participe de la visée descriptive/évaluative des rapports éducatifs. Mais il témoigne aussi de la porosité du discours des éducateurs avec le discours clinique «psy», qui fait partie de son interdiscours. Nous avons ainsi constaté que des patrons différents pouvaient participer du même fonctionnement discursif, ou qu'un même patron pouvait participer de plusieurs fonctionnements (voir Née, Sitri \& Veniard, 2014 et tableau 2 en annexe). Par ailleurs, utilisant les fonctions «topographiques » ou «topologiques » ${ }^{8}$ (Lamalle \& Salem, 2002;

7. On parle d'associations notionnelles quand les catégories sont d'ordre sémantico-pragmatique : «évolution» ou «capacité» par exemple.

8. La topographie textuelle peut se définir par le recours à un ensemble d'outils de visualisation et de lecture permettant de localiser précisément des unités textuelles ou un phénomène statistique concernant des unités textuelles sur un 
Mellet \& Barthélémy, 2009) du Trameur, nous avons pu montrer comment un fonctionnement discursif pouvait s'actualiser plus particulièrement dans une «partie » du texte, participant ainsi à la construction du texte (voir Née, Sitri \& Veniard, 2014; Fleury, Née, Sitri \& Veniard, à paraitre).

La notion de «routines» nous permet de rendre compte de cette association forme/fonction. Nous proposons de la définir de la façon suivante :

Une routine discursive consiste en la mise en relation de séquences linguistiques récurrentes, partiellement figées (i.e. les patrons définis plus haut) avec des déterminations discursives et des fonctions textuelles propres à un genre ou une sphère d'activité.

Notre perspective n'est pas fonctionnaliste, dans la mesure où nous ne cherchons pas à établir un relevé exhaustif des fonctions et des formes qui leur sont associées. Dans le cas des écrits professionnels sur lesquels nous avons fait porter nos recherches, ces routines peuvent être envisagées comme des ressources fabriquées par les scripteurs et à leur disposition, qui à la fois facilitent le processus d'écriture et participent de la construction d'une identité professionnelle tout en révélant des représentations propres au secteur. On peut d'ailleurs se demander si une telle définition ne caractérise pas les genres professionnels dans leur ensemble.

On voit aussi en quoi notre définition, si elle se rapproche sur le plan formel de la notion de «motif» proposée par Mellet (par exemple, Mellet \& Barthelemy, 2009; Mellet \& Longrée, 2012, p. 717) ${ }^{9}$, s'en distingue sur le plan fonctionnel. Pour ces auteurs, en effet, le motif est essentiellement envisagé dans sa fonction textuelle, par exemple des fonctions cohésive, résomptive, de structuration temporelle de la narration, de progression de l'intrigue, etc. À travers ces fonctions, le

corpus textuel, le texte étant alors considéré comme une structure ordonnée (voir Lamalle \& Salem, 2002; Mellet \& Barthelemy, 2009).

9. La notion de motif est définie par Mellet et Longrée (2012) comme «une nouvelle unité textuelle, qui intégrant formes graphiques, lemmes, catégories grammaticales, patrons syntaxiques et, éventuellement, schèmes métriques ou prosodiques, permet de traiter à la fois l'imbrication hiérarchique de ces différents niveaux linguistiques, leur association syntagmatique dans la chaîne linéaire des énoncés et leur récurrence structurante au niveau macrotextuel» (p. 717). 
motif participe à la structuration du texte. La notion de routine discursive, elle, vise à articuler des formes linguistiques à des déterminations d'ordre discursif (généricité et interdiscursivité notamment) et social. Les propositions de Legallois (2012) sont plus proches de notre perspective dans la mesure où celui-ci suggère que certains «motifs » peuvent remplir des fonctions argumentatives nées des besoins d'expression des locuteurs. On renvoie à son analyse de «les plus $+\mathrm{Adj}$ » et sa fonction de désignation de classes sociologiques dans un corpus de débats parlementaire (Legallois, 2012, p. 46). La dénomination de «routines » nous permet également d'envisager les patrons que nous avons identifiés du point de vue de la production («routines d'écriture»).

\section{Parcours méthodologique}

Nos travaux nous ont amenées à mettre au jour une série de routines discursives (voir Née, Sitri \& Veniard, 2014; Née, Sitri \& Fleury, 2014; Fleury, Née, Sitri \& Veniard, à paraitre), qui sont présentées en annexe. Pour illustrer notre propos, en particulier sur le plan méthodologique, nous présentons deux exemples de routines discursives ${ }^{10}$ : la première engage une unité lexicale (cadre) et une construction prépositionnelle (dans le cadre) tandis que la seconde se joue au niveau syntaxique (propositions coordonnées et modalité verbale). Il s'agit de deux routines de nature différente, mais qui toutes les deux mettent en jeu, comme nous le verrons, des «thèmes » centraux dans le discours des éducateurs.

La démarche suivie est précisée dans Née, Sitri \& Veniard (2014). Précisons simplement ici que des patrons potentiels sont identifiés à partir de l'inventaire des segments répétés (voir tableau 1 en annexe). Un retour au texte permet de dire si les segments répétés sont effectivement des patrons. Ensuite, ces derniers sont analysés en tant que routines potentielles. Si une ou plusieurs fonctions sont mises au jour, nous considérons qu'il s'agit d'une routine. Nous ne poursuivons pas, à l'heure actuelle, les objectifs d'un repérage qui serait 1) exhaustif et 2) automatique. À ce stade de la recherche, notre démarche est heuristique, dans la perspective de l'analyse du discours.

10. La nature des routines les rend difficiles à quantifier. On pourrait envisager une évaluation du degré de figement des textes du corpus à partir des segments répétés, à l'instar de ce qui est proposé par Salem (1987). 


\section{1. "Dans le cadre + spécification »}

Dans le corpus des états finaux des rapports (voir supra, note 3), la forme cadre se manifeste par une présence relativement importante (51 occurrences), compte tenu de la petite taille du corpus (44 178 occurrences). L'examen des inventaires de fréquences et de segments répétés permet de relever non seulement la présence d'un champ dérivationnel (encadrement, cadrer, recadrer, cadrage, recadrage), avec cependant des indices de fréquences très faibles $(<2)$, mais surtout l'expression dans le cadre, qui représente la moitié des occurrences (voir infra, tableau en annexe).

La saillance de cette forme est aussi signalée par un certain nombre de corrections qui apparaissent sur les différents états de rédaction des rapports et qui portent précisément sur ce champ dérivationnel ou font intervenir le mot cadre. En particulier, à plusieurs reprises, «dans le cadre» vient remplacer une autre préposition ou locution prépositionnelle :

(1)

Depuis le mois d'août 2009, Mme GILLOUARD rencontre Clara en entretiens mensuels. (État 1) $\rightarrow$ Depuis le mois d'août 2009, Mme GILLOUARD, psychologue, rencontre Clara dans le cadre d'entretiens mensuels. (État 2) (Dossier Clara)

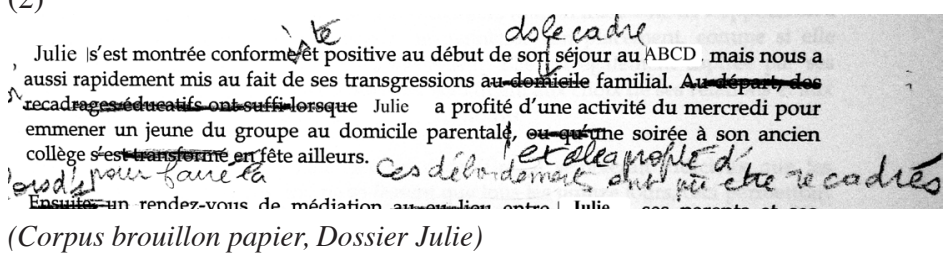

La haute fréquence de l'expression dans le cadre, son caractère relativement figé, la saillance de la forme cadre, enfin l'importance dans le discours des éducateurs de la notion de cadre (voir Cambon, 2009) nous ont amenées à poser l'hypothèse de l'existence d'une routine.

Adoptant une démarche comparative, consistant à étudier les réalisations et les contextes d'emploi de cette locution dans plusieurs genres (outre les rapports éducatifs, l'article scientifique ${ }^{11}$ et la dépêche de

11. Sous-corpus constitué à partir du corpus en ligne Scientext. Nous remercions ici Agnès Tutin, Olivier Kraif et Achille Falaise pour leur aide dans la consultation de ces données. 
presse ${ }^{12}$ ), nous avons pu faire apparaitre quelques spécificités propres au corpus des rapports éducatifs (Née \& Sitri, en préparation). Nous n'évoquerons ici que les particularités du point de vue sémantique. Ainsi, étant donné que la locution «dans le cadre» est généralement suivie d'un nom renvoyant à un espace abstrait étroitement lié avec la sphère d'activité considérée, dans les rapports éducatifs, les catégories de référents représentés, peu variés, sont de fait étroitement liés au travail social : il peut s'agir du type de mesure et du cadre institutionnel-légal dans lequel s'inscrit intervention de l'éducateur («dans le cadre du placement familial séquentiel», «dans le cadre de l'enquête sociale»...); des modalités ou des circonstances de la prise en charge par le service («dans le cadre de sa prise en charge par notre service», «dans le cadre d'entretiens individuels») ou encore du cadre dans lequel s'effectue l'observation («suite à des difficultés repérées dans le cadre du collège ceci à raison d'une séance par semaine», "nous avons organisé dans le cadre du service des rencontres entre Madame Ucar et Houria»).

Dans ce dernier cas, on voit que le syntagme le service peut également référer au lieu où se déroulent les entretiens. En effet, dans le corpus étudié, la locution «dans le cadre» admet à sa suite des noms de lieu, produisant des emplois peu naturels comme : «Monsieur Seyvet se promène avec sa fille dans le cadre du centre commercial proche de son domicile un samedi sur deux pendant quelques heures.»

Dans ce type d'emploi, il n'est plus question explicitement de la prise en charge mais du récit et de la description d'actions menées par la famille - le père, le plus souvent - à l'intérieur, «dans le cadre» de cette prise en charge. Étant donné cette spécialisation de l'emploi de la locution dans le genre considéré, nous avons donc formulé l'hypothèse, que «dans le cadre» construit le nom qui suit, même quand c'est un nom de lieu, en champ/domaine d'observation : le réel est ainsi «découpé» en cadres dans lequel s'effectue l'observation/évaluation du comportement des enfants confiés au service et de leur famille ${ }^{13}$. Cet emploi, qui peut sembler au premier abord contre-intuitif, n'est pas remarquable

12. Corpus réuni par Marine Damiani (Modyco) que nous remercions.

13. On peut également dire, comme le souligne un des relecteurs anonymes de l'article, que dans DET cadre construit le $\mathrm{N}$ qui suit en $\mathrm{N}$ d'activité, même quand c'est un $\mathrm{N}$ locatif, d'où l'effet d'étrangeté avec «se promener» qui demande un locatif. 
par sa fréquence mais par ce qu'il permet de révéler. En effet, étant donné la dimension narrative/descriptive du rapport éducatif, on attendrait plutôt des circonstants temporels et locatifs comme «au collège», «dans le centre commercial» ou «dans le service». L'expression «dans le cadre» introduit un autre effet de sens, lié à la visée évaluative qui demande un «cadre», «une grille». Se manifeste ici la double visée des rapports éducatifs, dans lesquels la description d'une situation est au service de l'évaluation: les personnes et leur comportement sont évalués dans les différents lieux où elles évoluent... y compris ceux qui ne sont pas institutionnels. En d'autres termes, tout lieu est potentiellement un lieu d'observation, tout espace dans lequel évolue le jeune ou sa famille devient potentiellement cadre.

On retrouve alors dans cet emploi la double valeur du «cadre» dans les discours des éducateurs analysés par Cambon (2009) : le cadre légal de l'intervention d'une part - les éducateurs sont «cadrés» par les textes de loi - mais aussi le cadre imposé par la norme ou par la loi, qui va servir d'étalon inconscient aux évaluations des comportements et des situations, et qu'ils ont pour mission d'«imposer» à ceux dont ils s'occupent (en les «cadrant»). On voit ainsi que la routine discursive mise en place autour du patron dans le cadre s'inscrit dans la visée évaluative du genre des rapports éducatifs, mais peut également s'interpréter comme l'actualisation d'un thème signifiant dans le discours des éducateurs, en relation avec leur identité professionnelle.

\subsection{La routine " et peut + INF"}

Notre second exemple est un patron d'ordre syntaxique transpropositionnel construit sur une coordination : deux propositions indépendantes coordonnées par «et», dont la seconde contient le verbe pouvoir en verbe principal. Il se résume par : «et peut + INF», comme dans (3) et (4). Nous proposons dans ce qui suit une description très synthétique de son fonctionnement.

(3)

Elle est également autonome dans les jeux, et peut rester un long moment à jouer seule dans sa chambre.

(4)

C'est pourquoi aujourd'hui il a évolué dans ses réflexions et peut mesurer un peu plus l'importance d'une telle formation pour son avenir professionnel. 
Ce patron, complexe, est moins fréquent que «dans le cadre (18 cas, dispersés dans 10 dossiers rédigés par 5 scripteurs ou groupes de scripteurs différents). Saillant, le modal «pouvoir» figure dans des segments répétés : on compte 54 et 26 occurrences, respectivement, de «a pu» et «il peut» (cf. tableau 1 en annexe). Il peut prendre dans le corpus un sens évènementiel décrit par Sitri (2013), sur lequel on reviendra plus bas. C'est la fréquentation du corpus qui a permis de repérer la récurrence de la coordination précédant le modal et de mettre au jour le patron «et peut $+\mathrm{INF}$ ». Les énoncés concernés ont été relevés grâce à la fonction «Patron» du logiciel Le Trameur en exploitant des expressions régulières ${ }^{14}$. Une fois le patron repéré, encore faut-il identifier les fonctions discursives et textuelles qu'il sert pour pouvoir l'interpréter en tant que routine. L'interprétation a reposé sur trois éléments : le sens de pouvoir, le sens des verbes de chacune des deux propositions et le sens de la coordination.

Sitri (2013) a mis au jour une valeur de pouvoir propre au corpus et distincte des valeurs traditionnellement reconnues telles que la permission, la capacité et la possibilité. À partir de la valeur de sporadicité, mise au jour par Kleiber (1983), comme dans «Jean peut être odieux», que l'on peut gloser en «il arrive que Jean soit odieux», Sitri montre que, dans le corpus, un emploi combinant la capacité et la sporadicité est caractéristique. Cet emploi prend un sens spécifique que l'on peut paraphraser par «le procès a eu lieu et c'est notable». Cette valeur de sporadicité-notabilité, note l'auteur, peut venir illustrer un énoncé généralisant, enchainement susceptible de se figer dans la routine «et peut + INF».

L'articulation entre les deux propositions nait de la coordination, porteuse de sens. Plutôt que de multiplier les valeurs (conséquence, opposition...), Serbat (1990) propose de considérer que la coordination par «et» réalise une énonciation à double détente. P1 est énoncé et le locuteur se transportant au terme de $\mathrm{P} 1$, il donne un deuxième coup d'envoi énonciatif, une «relance énonciative» (Serbat, 1990, p. 28). Cette relance se manifeste par un changement de type de verbes :

- en P1 : verbe d'état + adjectif/adverbe (être autonome, ancré, bien, redevenir accessible, se sentir bien/mal) ou des construc-

14. Recherche de 6 items, dont le lemme POUVOIR : [et POUVOIR *****], [et * POUVOIR ****] et [et .** POUVOIR ****]. 
tions attributives (être en demande affective, faire confiance, s'installer, passer, évoluer dans ses réflexions, avoir des souvenirs).

- en P2 : pouvoir + discuter, faire un récit, dire, changer d'avis, nouer une relation, être en relation, imaginer, mesurer l'importance, rester à jouer...

En P1, on relève en grande partie des verbes d'état ou des locutions verbales d'états psychologiques, alors qu'en P2, on observe des verbes de parole et d'attitudes verbales ou des verbes d'action. La proposition $\mathrm{P} 1$, générale, est souvent un jugement sur un état du sujet grammatical asserté par le scripteur; P2 constitue une description d'un POUVOIR FAIRE, c'est-à-dire l'énoncé d'une capacité possédée/acquise par l'enfant évalué ou d'un évènement notable le concernant. Les énoncés suivent le schéma pragmatique suivant : JUGEMENT d'ÉTAT - CAPACITÉ et/ou ÉVÈNEMENT NOTABLE. La principale fonction du patron est la preuve par l'exemple, ce qui fait de lui une routine argumentative. Ainsi, dans (5),

Marjorie présente une grande autonomie dans les gestes du quotidien : habillage, repas...

Elle est également autonome dans les jeux, et peut rester un long moment à jouer seule dans sa chambre.

la mention de la compétence «jouer seule dans sa chambre» constitue un exemple qui vient renforcer la thèse énoncée en P1, «l'enfant est autonome».

Dans cette configuration, le patron est une des actualisations possibles de la fonction «argument par l'exemple», ce qui peut s'inscrire dans une des prescriptions régulant ce type d'écrit, à savoir éviter les énoncés trop généraux et étayer ses jugements :

(6)

Thierry s'est réellement installé en famille d'accueil et a pu nouer une relation de confiance avec son assistante familiale.

Pour autant, il [le père] fait confiance à la famille d'accueil et a pu nous exprimer son accord et sa satisfaction concernant le suivi de Nathalie par notre service dans un récent courrier. 
Comme pour «dans le cadre», on voit là apparaitre le rôle assigné à l'éducateur : observer et évaluer. Il faut noter, toutefois, que la valeur argumentative ne rend pas compte de l'intégralité des fonctions du patron. On peut relever des fonctions telles que la chronologie (8) ou une relation consécutive (9), marginales du point de vue du nombre d'occurrences (2 au plus).

Il redevient accessible assez rapidement et peut en discuter librement.

(9)

De nouveau, il est nécessaire d'envisager un accueil relais tant Emmanuel, pris dans ses angoisses, se sent mal et ne peut imaginer rester en famille d'accueil.

Ces deux exemples, différents par leur fréquence, sont révélateurs de la variété des routines, du syntagme aux propositions, un figement plus ou moins prononcé, mais également de ce qui constitue leur spécificité, à savoir l'articulation d'un patron formel à des fonctions ou déterminations hétérogènes que l'on peut saisir à travers le genre.

\section{Conclusion}

Si le genre est une catégorie bien établie en analyse du discours, ce n'est pas le cas de la routine. Le genre tire son intérêt de son hétérogénéité : c'est une catégorie qui permet l'interface entre des niveaux de détermination différents, aussi bien le linguistique, que le textuel, le pragmatique et l'interdiscursif. La routine discursive, telle que nous l'entendons, est également une catégorie d'interface qui permet de mettre en regard formes linguistiques et interprétation. Cette propriété en fait selon nous une catégorie d'analyse opératoire pour une analyse de discours soucieuse d'appuyer l'interprétation sur des formes. Des recherches futures devraient permettre de valider (ou pas) son caractère opératoire dans d'autres genres de discours et dans d'autres sphères d'activité.

La catégorie des routines fait également émerger la question de la subjectivité du scripteur. En effet, l'adossement de notre réflexion sur les routines dans des écrits d'éducateurs spécialisés à une approche génétique, dans le cadre plus global du projet ANR Écritures (Resp. G. Cislaru, Université Sorbonne Nouvelle-Paris 3, 2010-2014) (voir supra, note 3) a déplacé notre regard de l'écrit produit vers le processus d'écriture, ce qui constitue un positionnement inhabituel en analyse de 
discours. La notion de routine permet ainsi de rattacher des modifications effectuées par des scripteurs individuels à des fonctionnements discursifs plus généraux qui viennent les expliquer et qui semblent s'imposer aux rédacteurs. Une telle notion pourrait dès lors ouvrir la voie à une exploration des relations entre processus d'écriture et analyse de discours.

\section{RÉFÉRENCES BIBLIOGRAPHIQUES}

BAKHTine, Mikhail. (1984) [1952-1953]. Les genres du discours. Dans Esthétique de la création verbale. Paris : Gallimard.

Barthélémy, Jean-Pierre \& Mellet, Sylvie. (2009). La topologie textuelle : légitimation d'une notion émergente. Lexicometrica, numéro spécial «Topographie et topologie textuelle», 1-15.

BerRendonner, Alain. (2008). Pour une praxéologie des parenthèses. Verbum, $X X X(1), 5-23$.

Borzeix, Anni \& Fraenkel, Béatrice. (2001). Langage et travail. Paris : CNRS éditions.

BRANCA-Rosoff, Sonia. (1990). Conventions d'écriture dans la correspondance des soldats. Mots, 24, 21-36.

Branca-Rosoff, Sonia. (1997). Modèles de locutionnarité et effets de figement dans le discours politique de l'an II. Dans P. Fiala, P. Lafon \& M.-F. Piguet, (dir.), La locution : entre lexique, syntaxe et pragmatique (p. 285-293). Paris : Klincksieck.

Branca-Rosoff, Sonia. (1999). Types, modes et genres entre langue et discours. Langage et Société, 87, 5-24.

Bronckart, Jean-Paul. (1997). Activité langagière, textes et discours. Pour un interactionnisme socio-discursif. Paris : Delachaux et Niestlé.

Corminboeuf, Gilles. (2013). Négation et asyndète. Dans J. François, P. Larrivée, D. Legallois \& F. Neveu (dir.), La linguistique de la contradiction (p. 233-242). Berne : P. Lang.

Cаmbon, Laurent. (2009). L'identité professionnelle des éducateurs spécialisés. Une approche par les langages. Rennes: Presses de l'École des Hautes Études en Santé Publique.

Coulmas, Florian (dir.). (1981). Conversational Routine. La Haye : Mouton.

Delcambre, Pierre. (1993). Écriture/écrits professionnels : quelles interactions au travail? Dispositif d'écriture et sujets en milieu de travail. Cahiers langage et travail, 6, 63-78.

Fiala, Pierre, Habert, Benoit, Lafon Pierre \& Pineira, Carmen. (1987). Des mots aux syntagmes, figements et variations dans la Résolution générale du congrès de la CGT de 1978. Mots, 14, 45-87. 
Fiala, Pierre. (1987). Pour une approche discursive de la phraséologie. Remarques en vrac sur la locutionnalité et quelques points de vue qui s'y rapportent, sans doute. Langage et Société, 42, 28-48.

Fleury, Serge. (2007). Le Métier Textométrique : Le Trameur, Manuel d'utilisation. Disponible en ligne sur le site de l'Université Sorbonne Nouvelle-Paris 3, Centre de Textométrie : <http://tal.univ-paris3.fr/ trameur/> (consulté le 29 mars 2016).

Fleury, Serge. (2013). Le Trameur. Propositions de description et d'implémentation des objets textométrique. Disponible en ligne sur le site de l'Université Sorbonne Nouvelle-Paris 3, Centre de Textométrie : $<$ www.tal.univ-paris3.fr/trameur/trameur-propositions-definitionsobjets-textometriques.pdf $>$ (consulté le 29 mars 2016).

Fleury, Serge, Née, Émilie, Sitri, Frédérique \& Veniard, Marie. (À paraitre). Routines discursives et textualité dans des écrits professionnels. Corpus, 15.

Groupe de Fribourg. (2012). Grammaire de la période. Berne : P. Lang. Gorcy, Gérard, Martin, Robert, Maucourt, Jacques \& Vienney, Roland (1970). Le traitement des groupes binaires. Cahiers de Lexicologie, 17, 15-46.

Heiden, Serge \& Lafon, Pierre. (1998). Cooccurrences. La CFDT de 1973 à 1992. Dans P. Fiala \& P. Lafon (dir.), Des mots en liberté. Mélanges Maurice Tournier (t. 1, p. 65-83). Fontenay-aux-Roses : ENS Éditions.

Hyland, Ken. (2010). Metadiscourse: Mapping Interactions in Academic Writing. Nordic Journal of English Studies, 9(2), 125-143.

KLEIBER, Georges. (1983). L'emploi «sporadique» du verbe pouvoir en français. Recherches linguistiques, 8, 181-203.

KrIeg-Planque, Alice. (2013). Analyser les discours institutionnels. Paris : A. Colin.

Krishnamurthy, Ramesh (dir.). (2004). English Collocation Studies: The OSTI Report. Londres/New York : Continuum.

LAFON, Pierre. (1981). Analyse lexicométrique et recherche des cooccurrences. Mots, 3, 95-148.

Lamalle, Cédric \& Salem, André. (2002). Types généralisés et topographie textuelle dans l'analyse quantitative des corpus textuels. Dans A. Morin \& P. Sébillot (dir.), Actes des $\sigma^{e}$ Journées d'analyse statistique des données textuelles (p. 403-411). Saint-Malo. Disponible en ligne sur le site de l'Université Sorbonne Nouvelle-Paris 3 : <http://lexicometrica. univ-paris3.fr/jadt/jadt2002/PDF-2002/lamalle_salem.pdf> (consulté le 29 mars 2016).

Lebart, Ludovic \& Salem, André. (1994). Statistique textuelle. Paris : Dunod. 
Legallois, Dominique. (2012). La colligation : autre nom de la collocation grammaticale ou autre logique de la relation mutuelle entre syntaxe et sémantique? Corpus, 11. Disponible en ligne sur <http://corpus.revues. org/2202> (consulté le 18 septembre 2014).

Maingueneau, Dominique. (2004). Retour sur une catégorie : le genre. Dans J.-M. Adam, J.-B. Grize \& M. Ali Bouacha (dir.), Texte et discours : catégories pour l'analyse (p. 107-118). Dijon : Éditions universitaires de Dijon.

Martinez, William. (2003). Contribution à une méthodologie de l'analyse des cooccurrences lexicales multiples dans les corpus textuels (Thèse de doctorat en sciences du langage non publiée). Université Sorbonne Nouvelle-Paris 3, Paris.

MAYAFFre, Damon. (2008a). Quand «travail», «famille», «patrie» cooccurrent dans le discours de Nicolas Sarkozy. Étude de cas et réflexion théorique sur la co-occurrence. Dans S. Heiden \& B. Pincemin (dir.), Actes des $9^{e}$ Journées internationales d'analyse statistique des données textuelles - JADT 2008 (vol. 2, p. 811-822). Lyon : Presses universitaires de Lyon. Disponible en ligne sur le site de l'Université Sorbonne Nouvelle-Paris $3:<$ http://lexicometrica.univ-paris3.fr/jadt/jadt2008/ pdf/mayaffre.pdf $>$ (consulté le 29 mars 2016).

MAYAFFre, Damon. (2008b). De l'occurrence à l'isotopie. Les co-occurrences en lexicométrie. Sémantique et Syntaxe, 9, 53-72.

Mellet, Sylvie \& Salem, André (dir.). (2009). «Topographie et topologie textuelles », numéro spécial de Lexicométrica. Disponible en ligne sur le site de l'Université Sorbonne Nouvelle-Paris $3:<\mathrm{http} / / /$ lexicometrica. univ-paris3.fr/numspeciaux/special9.htm> (consulté le 8 déc. 2014).

Mellet, Caroline \& Sitri, Frédérique. (2010). Nom de genre et institutionnalisation d'une pratique discursive : le cas de l'interpellation parlementaire et du signalement d'enfant en danger. Dans F. Neveu et coll. (dir.), Actes du CMLF 2010 - 2 $2^{e}$ Congrès mondial de linguistique française (p. 797-810). EDP Sciences. Disponible en ligne sur <http://dx. doi.org/10.1051/cmlf/2010175>.

Mellet, Caroline, Rinck, Fanny \& Sitri, Frédérique. (2013). Hétérogénéité des genres. Pratiques («Théories et pratiques des genres»), 157 158, 47-59.

Mellet, Sylvie \& Longrée, Dominique. (2012). Légitimité d'une unité textométrique : le motif. Dans A. Dister, D. Longrée \& G. Purnelle (dir.), Actes des $11^{e}$ Journées internationales d'analyse statistique des données textuelles - JADT 2012 (p. 715-728). Liège. Disponible en ligne sur $<$ http://lexicometrica.univ-paris3.fr/jadt/jadt2012/Communications/ Mellet,\%20Sylvie\%20et\%20al.\%20-\%20Legitimite\%20d'une\%20 unite\%20textometrique.pdf> (consulté le 29 mars 2016). 
Née, Émilie, MacMurray, Erin \& Fleury, Serge. (2012). Textometric Explorations of Writing Processes: A Discursive and Genetic Approach to the Study of Drafts. Dans Actes des $11^{e}$ Journées internationales d'analyse statistique des données textuelles - JADT 2012. Liège. Disponible en ligne sur <http://lexicometrica.univ-paris3.fr/jadt/jadt2012/ Communications/Nee,\%20Emilie\%20et\%20al.\%20-\%20Textometric $\%$ 20Explorations\%20of\%20Writing\%20Processes.pdf> (consulté le 29 mars 2016).

Née, Émilie \& Sitri, Frédérique. (En préparation). Dans det. Cadre (+ spécification) : de la locution à la routine discursive.

Née, Émilie, Sitri, Frédérique \& Fleury, Serge (2014). L'annotation du pronom «nous » dans un corpus de rapports éducatifs. Objectifs, méthodes, résultats. Dans É. Née, M. Valette, J.-M. Daube \& S. Fleury (dir.), Actes des $12^{e}$ Journées internationales d'analyse statistique de données textuelles - JADT 2014. Paris. Disponible en ligne sur <http:// lexicometrica.univ-paris3.fr/jadt/jadt2014/01-ACTES/41-JADT2014. pdf> (consulté le 29 mars 2016).

Née, Émilie, Sitri, Frédérique \& Veniard, Marie. (2014). Pour une approche des routines discursives dans les écrits professionnels. Dans F. Neveu et coll. (dir.), Actes du CMLF 2014 - $4^{e}$ Congrès mondial de linguistique française (vol. 8, p. 2113-2124). EDP Sciences. Disponible en ligne sur <http://dx.doi.org/10.1051/shsconf/20140801195>.

PÈne, Sophie. (1994). Analyse de postes, bilan d'entretiens, écritures de procédures. Un «prêt-à-écrire» pour inscrire l'activité. Éducation permanente, 120, 39-57.

Plane, Sylvie, Alarmagot, Denis \& Lebrave, Jean-Louis. (2010). Temporalité de l'écriture et rôle du texte produit dans l'activité rédactionnelle. Langages, 177, 7-28.

Salem, André. (1987). Pratique des segments répétés. Essai de statistique textuelle. Paris : Klincksieck.

SAndor, Agnes. (2007). Modeling Metadiscourse Conveying the Author's Rhetorical Strategy in Biomedical Research Abstracts. Revue française de linguistique appliquée, 12, 97-97.

Serbat, Guy. (1990). Et «jonctif» de propositions : une énonciation à double détente. L'Information grammaticale, 46, 26-28.

SitRi, Frédérique (2013). Une lecture événementielle du verbe «pouvoir» dans des rapports de travailleurs sociaux. Dans D. Londei, S. Moirand, S. Reboul-Touré \& L. Reggiani (dir.), Dire l'événement. Langage, mémoire, société (p. 73-84). Paris : Presses Sorbonne Nouvelle.

Swales, John. (1990). Genre Analysis: English in Academic and Research Settings. Cambridge : Cambridge University Press. 
Traverso, Véronique. (1996). La conversation familière. Analyse pragmatique des interactions. Lyon : Presses universitaires de Lyon.

Tutin, Agnès. (2007). Autour du lexique et de la phraséologie des écrits scientifiques. Revue française de linguistique appliquée , 12, 5-14.

Tutin, Agnès. (2013). La phraséologie transdisciplinaire des écrits scientifiques : des collocations aux routines sémantico-rhétoriques. Dans A. Tutin \& F. Grossmann (dir.), L'écrit scientifique : du lexique au discours. Autour de Scientext (p. 27-43). Rennes : Presses universitaires de Rennes. 


\section{ANNEXES}

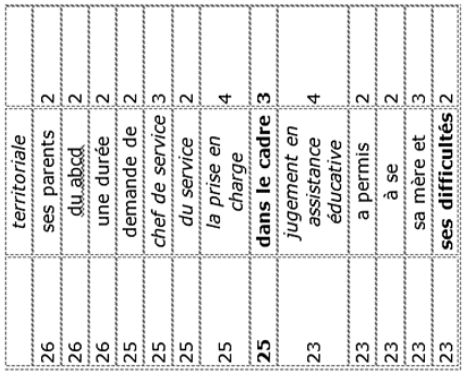

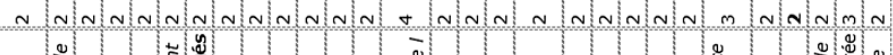

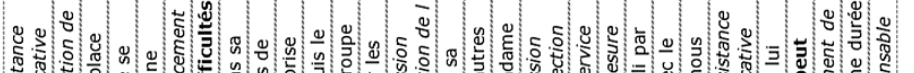

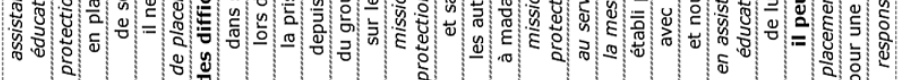

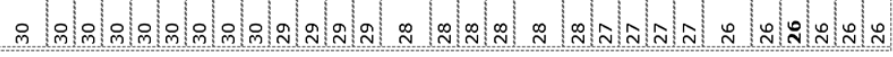
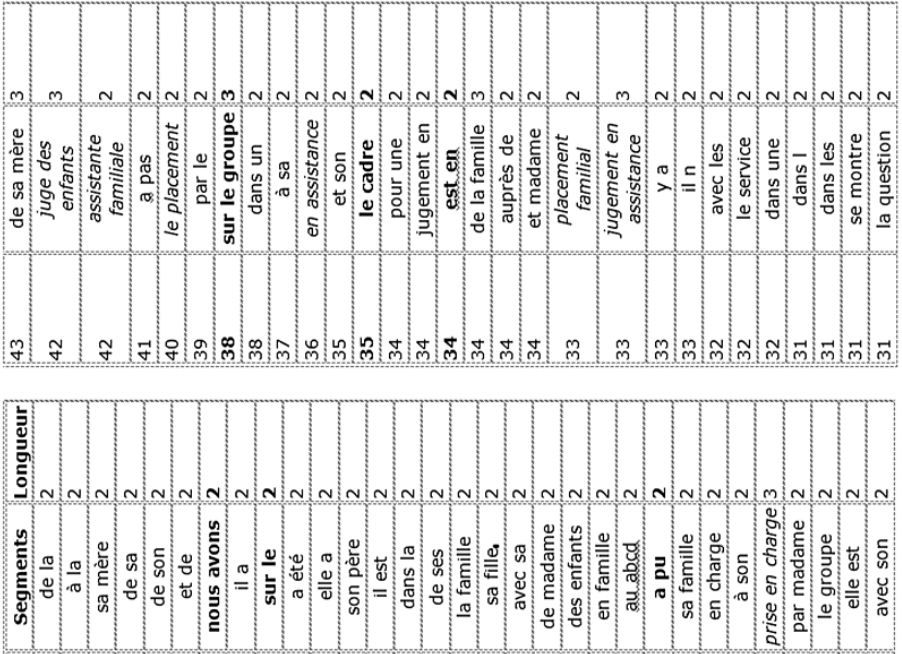

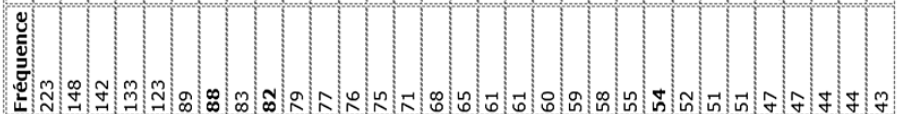

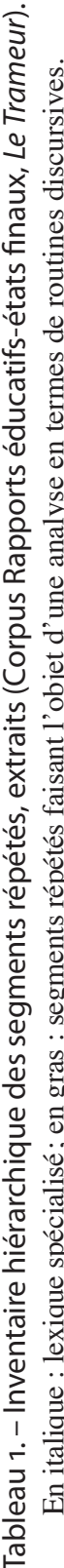




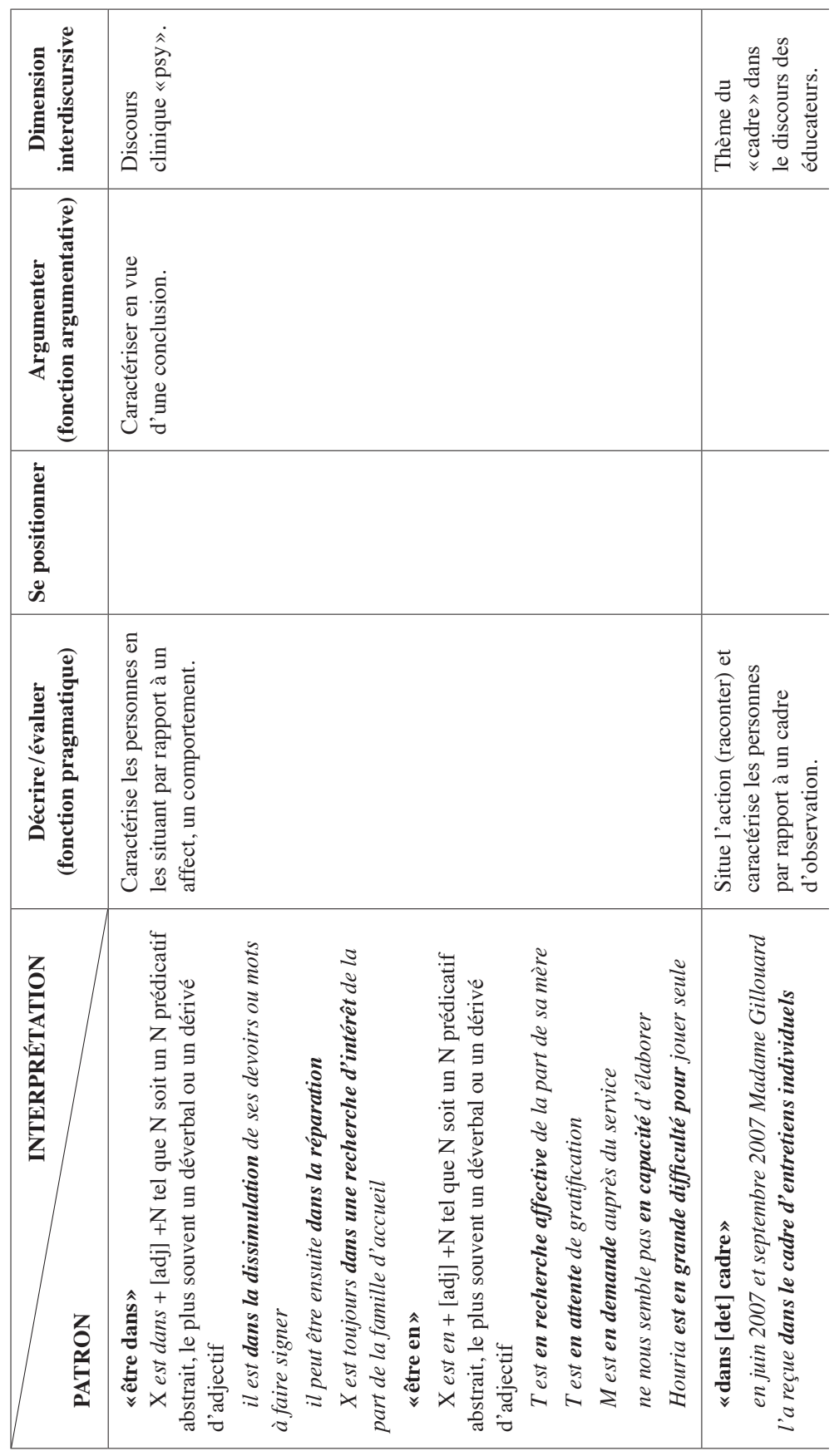




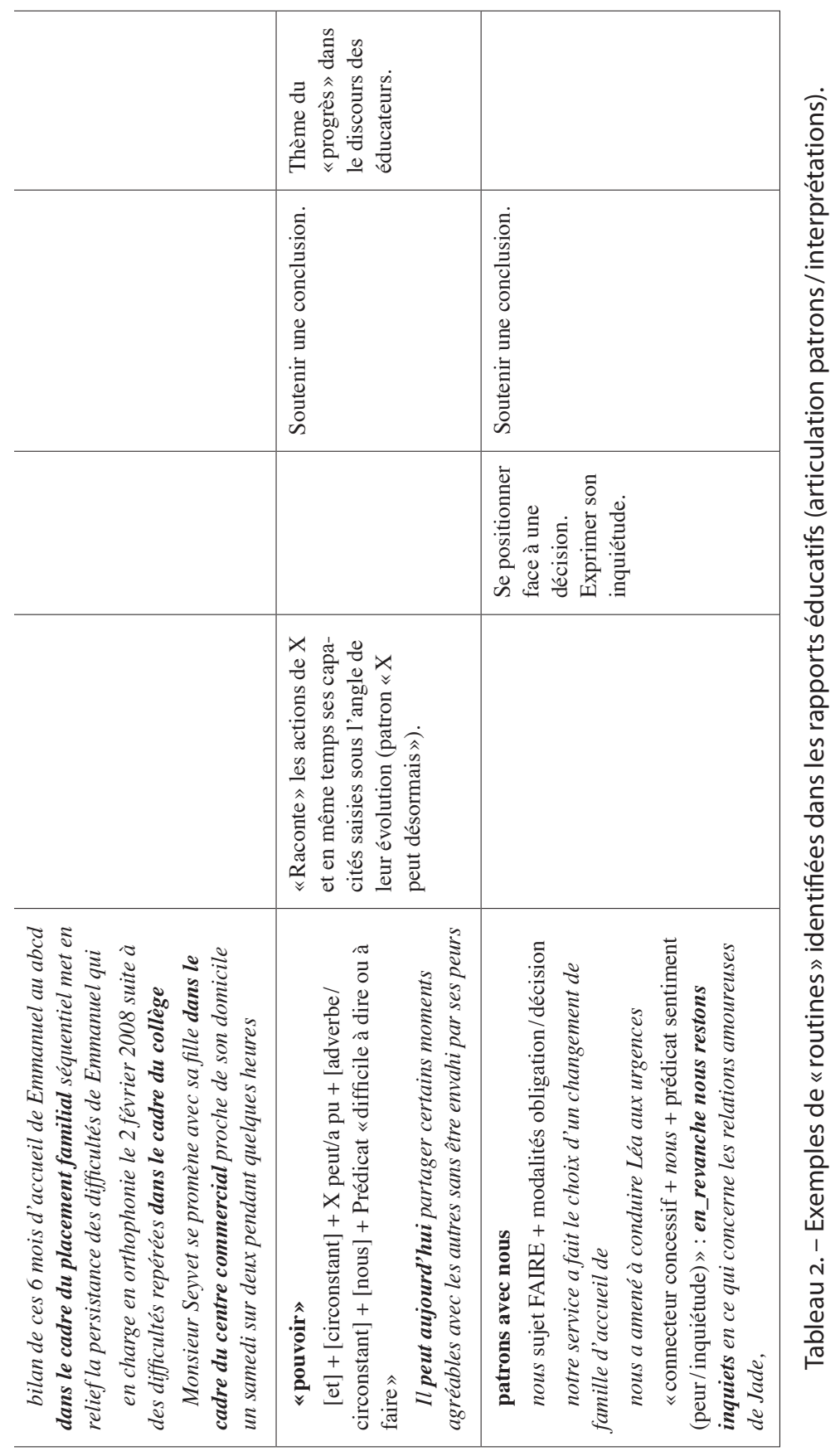

